\title{
A ROBUST NUMERICAL FRAMEWORK TO THE ANALYSIS OF MATERIAL FAILURE OF FIBRE REINFORCED SOFT TISSUE.
}

\author{
S. Blanco (1), J.M. Goicolea (1), C. Polindara (1) \\ 1. Department of Continuum Mechanics and Theory of Structures, Polytechnic \\ University of Madrid, Professor Aranguren s/n, 28040 Madrid,
}

\section{Introduction}

In this work, robustness and stability of continuum damage models applied to material failure in soft tissues are addressed. In the implicit damage models equipped with softening, the presence of negative eigenvalues in the tangent elemental matrix degrades the condition number of the global matrix, leading to a reduction of the computational performance of the numerical model.

Two strategies have been adapted from literature to improve the aforementioned computational performance degradation: the IMPL-EX integration scheme [Oliver,2006], which renders the elemental matrix contribution definite positive, and arclength-type continuation methods [Carrera,1994], which allow to capture the unstable softening branch in brittle ruptures. The IMPL-EX integration scheme has as a major drawback the need to use small time steps to keep numerical error below an acceptable value. A convergence study, limiting the maximum allowed increment of internal variables in the damage model, is presented. Finally, numerical simulation of failure problems with fibre reinforced materials illustrates the performance of the adopted methodology.

\section{Methods}

Mechanical behaviour of biological fibre-reinforced materials has been reproduced by using the constitutive law proposed by [Gasser,2006]. Each term of the energy density function (related to the incompressible matrix and each of the existing families of fibres) is affected by a damage variable, whose evolution is given by imposing that the total dissipation of the model depends on a material parameter. This approach is based on the smeared crack models and seeks to ensure the mesh-size independence of the model.

In presence of softening, constitutive operators can have negative eigenvalues and therefore degrade the condition number of the global matrix. The IMPL-EX integration scheme used renders this operator definite positive. This algorithm has two simultaneous integration schemes: a standard implicit scheme and an explicit scheme. In the explicit scheme, the internal variables of the damage model are calculated by extrapolating the values of these variables in previous time steps obtained with the implicit scheme. The fundamental feature of this method is that the integration of the stresses is performed with the internal variables obtained with the explicit method.

\section{Results}

Numerical robustness of the adopted model has shown its suitability to capture the whole equilibrium curve, including the softening branch, as is shown in Figure 1.

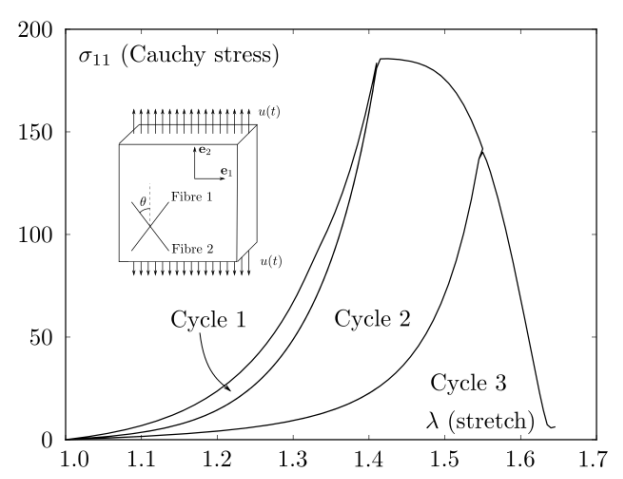

Figure 1: Uni axial tension test. Equilibrium curve.

However, integration errors should be controlled because IMPL-EX scheme provides smaller accuracies for large time steps in comparison with the ones obtained with the purely implicit integration procedure. This error control has been implemented by limiting the maximum variation of the internal variables in the damage model.

\section{Discussion}

Stability and robustness of the numerical models devoted to reproduce the material failure of fibrereinforced soft tissues have been addressed. The IMPL-EX integration scheme, along with suitable continuation arc-length methods and control error strategies, has proven to be a suitable numerical tool to capture the material failure associated with deformation softening.

\section{References}

E. Carrera, Computers and Structures, 50-2:217229, 1994.

Gasser et al, J. R. Soc. Interface, 3-6:15:35, 2006.

J. Oliver et al, Computer Methods in Applied Mechanics and Engineering, 195:7093-7114, 2006. 